\title{
e-Phaïstos
}

e-Phaïstos Revue d'histoire des techniques / Journal of the history of technology

II-2 | 2013

Les sources de l'histoire des techniques (2)

\section{Du danger des sources écrites en Histoire des techniques}

The Danger of Written Sources in the History of Technology

\section{Anne-Françoise Garçon}

\section{OpenEdition}

Journals

Édition électronique

URL : http://journals.openedition.org/ephaistos/6143

DOI : 10.4000/ephaistos.6143

ISSN : 2552-0741

Éditeur

IHMC - Institut d'histoire moderne et contemporaine (UMR 8066)

Édition imprimée

Date de publication : 15 décembre 2013

Pagination : 10-27

ISSN : 2262-7340

\section{Référence électronique}

Anne-Françoise Garçon, « Du danger des sources écrites en Histoire des techniques », e-Phaïstos [En ligne], II-2 | 2013, mis en ligne le 12 décembre 2019, consulté le 14 décembre 2019. URL : http:// journals.openedition.org/ephaistos/6143 ; DOI : 10.4000/ephaistos.6143 


\title{
Du danger des sources écrites en Histoire des Techniques
}

\author{
Anne françoise Garçon \\ Équipe d'Histoire des Techniques / IHMC \\ CNRS-Université Paris 1 Panthéon- \\ Sorbonne
}

Poser la question des sources en Histoire des techniques, c'est s'intéresser à l'une des spécificités de ce champ historique. Je ne reviendrai pas dans cet article sur ce qui caractérise l'Histoire en tant que construction scientifique. Simplement parce que cela impacte directement le sujet traité, je rappellerai, en préliminaire, que l'approche historique, en tant qu'ordonnancement scientifique du passé, se distingue de l'approche mémorielle et de l'approche patrimoniale ${ }^{1}$. Le patrimoine relève de l'héritage. Il est ce qui, du passé, se transmet aux générations futures, qu'il soit ou non valorisable ${ }^{2}$; et les générations futures peuvent l'accepter ou le rejeter. La mémoire renvoie à l'élaboration temporelle individuelle et/ou collective ; elle est ce que le présent reçoit et peut accepter du passé; elle comporte obligatoirement des phases d'oublis et de réinterprétations ${ }^{3}$. L'Histoire, en regard, est une tentative scientifique d'interprétation, voire de découverte de l'organisation passée d'une communauté humaine ; elle relève de la volonté d'expliquer scientifiquement l'évolution de cette communauté, dans sa structure propre, dans sa relation avec les communautés environnantes, dans sa relation avec les milieux et environnements qu'elle a croisés, modifiés, au fil des temps, un désir de compréhension de l'influence des représentations, actions, organisations qu'elle a développées ou qu'elle développe, de l'impact de ce vécu collectif sur les conceptions du monde en vigueur si d'aventure elle existe encore. Ajoutons à ces définitions, un autre élément essen- tiel : toute communauté humaine structurée élabore un récit mémoriel et dispose d'un patrimoine technique, culturel, etc. susceptible d'être transmis aux générations à venir; mais une communauté humaine peut vivre des siècles, voire des millénaires, sans ressentir le besoin d'élaborer une science historique ${ }^{4}$. Contrairement à la narration mythique, le discours historique n'est pas indispensable à la construction sociétale. Son invention suit de peu, à la fin du XVIIe siècle, l'invention de la pensée scientifique moderne, et elle lui est coextensive. Diderot encore, au milieu du XVIII e siècle, lorsqu'il faisait de l'Histoire, la Philosophie et la Poésie, les trois grands domaines de l'entendement humain, rangeait l'Histoire, non du côté de la raison, domaine de la philosophie qui regroupait, outre la métaphysique générale, les trois sciences de dieu, de l'homme et de la nature, mais du côté de la Mémoire. Et il plaçait au sein de l'Histoire naturelle, l'histoire des usages de la nature : arts (c'est-à-dire techniques), métiers et manufactures 5 .

L'Histoire, bien évidemment, n'est pas la seule parmi les sciences à œuvrer à la reconstitution du passé et à sa compréhension. D'autres disciplines font également le travail, de plus en plus nombreuses, tant est devenue indispensable sur une planète désormais surchargée d'humains, la connaissance intime des milieux, de leurs occupations et ordonnancements passées. Parmi elles, l'archéologie, l'archéométrie, les sciences du paysage, les sciences de l'environnement bénéficient 
d'outils scientifiques innovants et remarquablement performants. Hélas, le travail pluridisciplinaire cède souvent le pas à la guerre de tranchées. Le positivisme de la preuve, syndrome facile à développer en sciences dures, refoule l'Histoire aux marges de l'interprétation historique. Avec des griefs clairement énoncés : l'Histoire, n'aurait pour seul domaine d'investigation, que les objets sociaux, du fait qu'elle travaille sur les documents écrits ${ }^{6}$; ses seuls intérêts seraient «la recherche des situations d'origine et la reconstitution de phases historiques » et elle n'aurait pour dynamique que celle définie par "les intentionnalités sociales unilatérales $^{7}$ »; enfin, en tant que science, elle ne serait pas fiable, puisque ses sources, écrites au premier chef, ne le sont pas $^{8}$.

Pourtant, l'histoire des techniques existe (encore) et s'occupe en priorité d'une matérialité qu'il n'est pas possible de réduire au social9. La relation homme-technique dispose d'une historicité qui lui est propre, et jouit d'une autonomie qui la rend discernable dans l'ordonnancement sociétal, au même titre que l'économique, le culturel, le religieux, le politique. Donc, oui, les techniques ont une histoire, et pas seulement une archéologie ; oui, cette histoire nécessite un travail approfondi sur les textes et la langue. Car les artefacts archéologiques, s'ils témoignent positivement de la matérialité, ne disent rien de la pensée technique, ni des modalités d'apprentissage et de transmission; et s'ils disent des transferts techniques, des choix techniques et des adéquations pratiques/cultures, c'est très peu ${ }^{10}$. L'histoire des techniques n'est pas une science annexe des sciences de conservation/restauration ou des sciences patrimoniales. Elle est un champ historique à part entière, doté de moyens d'investigations qui lui sont propres, que Lucien Febvre définissait en son temps dans les Annales ${ }^{11}$.

Lucien Febvre justement. Il rappelait, dans son Combat pour l'Histoire que c'est bien l'historien qui fabrique ses sources, et non l'inverse. S'il suffisait, disait-il en substance, pour construire l'histoire d'enfiler les faits comme des perles sur un collier, point besoin ne serait d'historiens de métier ${ }^{12}$. Et, nous aurions, dans les archives, des érudits polygraphes, intelligents et passionnés, qui, tels les généalogistes qui en sont désormais les principaux clients, reconstitueraient le passé antérieur, l'historique commun. Or, cela n'est pas. Les érudits existent bien sûr. Mais, ils n'épuisent pas la veine historique. L'historique est sans aucun doute la condition nécessaire à la connaissance du passé ; il n'en n'est pas la condition suffisante. Chacun comprend aujourd'hui, face la complexité des évènements, devant la largeur de la scène évènementielle et son épaisseur factuelle, qu'il ne suffit pas d'être informé pour comprendre et de connaître pour expliquer.

L'Histoire est une herméneutique ${ }^{13}$. Comme tout savoir raisonné, elle comporte sa théorie et sa pratique; elle est tout à la fois art et science, travaillant à l'objectivation du passé en usant de la topique conceptuelle comprendre/expliquer/interpréter ${ }^{14}$. Elle est aussi outil de compréhension du présent. Érudite, elle n'a pas pour objectif l'érudition. Discursive, elle n'a pas pour objectif la narration. Précise, elle n'a pour objectif la précision, mais le dévoilement, au sens où l'entendait Nietzsche, dévoilement des ressorts de l'action, dévoilement du jeu des acteurs, individuels et collectifs, et de leurs rôles. Informée, elle n'a pas pour objectif l'information, mais la compréhension. Contrairement au journaliste, l'historien cite ses sources. Il les cite pour que chacun puisse s'y référer pour juger de la qualité de l'interprétation proposée, de la validité de l'explication, pour que chacun aussi puisse juger de la qualité de la source proposée. Ainsi, il détermine ses limites en ouvrant la porte à la réfutation. Présenter ses sources historiques, n'est donc pas seulement déterminer les fondements analytiques et scripturaires de l'énoncé. C'est aussi poser les conditions de la réfutabilité, donner les moyens d'interprétations différenciées, complémentaires, divergentes, qui déboucheront sur de nouveaux dévoilements, l'approfondissement ou l'élargissement, voire la réfutation des compréhen- 
sions, des explications. Voilà pourquoi, toute écriture académique de l'histoire débute par une présentation des sources, et comporte leur analyse critique.

Lucien Febvre toujours, recommandait d'opérer la généalogie du sujet, au moment d'initier une recherche. Recommandation précieuse. Opérer la généalogie du sujet, c'est travailler à son historiographie, comprendre donc la manière dont le sujet a été abordé, analysé, compris, travaillé avant que l'historien ne décide lui-même de s'y intéresser. L'interrogation renvoie aux sources bien sûr, en se plaçant du côté de l'analyse critique, cette fois. Établir la généalogie du sujet, c'est d'abord comprendre la manière dont le substrat historique s'est constitué, ce qu'il comporte et de quoi l'historien décide de le constituer. Elle renvoie à la littérature aussi, sans confiner l'approche à l'historiographie. Établir la généalogie du sujet, c'est aussi examiner la manière dont les autres sciences humaines et sociales s'en sont emparés et l'ont travaillé, anthropologie, ethnologie, sociologie, tout particulièrement, voire philosophie. Cet examen généalogique comporte nécessairement, en complément, une part de recherche épistémologique, qui concerne cette fois les «idoles personnelles » 15 , mais aussi celles de «la tribu» et celles de la «place publique »16, pour reprendre les termes de Francis Bacon, de manière à les mettre le plus à distance possible. "Idoles personnelles " : d'où vient que je m'intéresse au sujet, quel est mon lien avec le sujet, où est cette part de subjectivité personnelle qui pourrait gêner mon appréciation, obérer ma faculté d'analyse; "Idoles de la tribu» : quelles sont, en général, et spécifiquement pour le champ dans lequel je travaille, et plus encore spécifiquement le sujet auquel je m'intéresse, les présupposés habituels, les habitus, les postulats. «Idoles de la place publique» : quels sont les regards habituellement portés sur le sujet, les interprétations faites, l'explication qu'en donne le sens commun, etc. Ce travail épistémologique aide à ne pas tomber dans les pièges d'une problématisation naïve, erronée, ou tout simple- ment surfaite, à anticiper aussi sur les objections que le lecteur ne manquera pas de faire, à partir de ce que lui dicte le sens commun, en l'aidant à construire une position critique vis-à-vis de ce qui lui a été inculqué, de bonne foi, ou à son insu, de le sortir en somme de sa position initiale de « wikipédiste ».

Dans ce cadre, je vais me focaliser sur l'une des «idoles» de notre tribu : la difficulté qu'a l'historien à mettre à distance la source écrite. J'entends ici, par source écrite, les sources imprimées ou manuscrites qui se donnent directement pour des énoncés techniques ${ }^{17}$. Car ce type de source, fréquemment convoqué lorsque l'on cherche à comprendre des techniques révolues ou tout simplement mal connues, représentent un danger.

Écrire n'est pas anodin. Tous, nous avons expérimenté la difficulté que représente le passage de l'oral à l'écrit. Mettre par écrit ses idées s'avère au mieux malaisé, au pire décevant, et dans tous les cas, cela suppose une expérience, un dépassement, et, bien au-delà des connaissances que l'on verbalise, une maîtrise. L'écriture, au plus ancien de son usage, est liée à la comptabilité, à la gestion des stocks, d'une part, au sacré, au religieux d'autre part. Mais pourquoi coucher par écrit, transcrire le déroulement d'une chaîne opératoire ? La nécessité première ne s'en est pas fait sentir. L'apprentissage et la transmission de l'immense bibliothèque de savoir-faire que s'est constituée l'humanité, qu'il s'agisse des savoir-faire appliquée à la nourriture : pêche, chasse, cueillette, connaissance des plantes et manière de les cultiver, de les conserver ; des savoir-faire liés à l'habillement: filage, tressage, tissage, feutrage, couture; des savoir-faire liés à la fabrication et au maniement des outils, des armes, à la fabrication et au maniement des moyens de déplacement: embarcations, brancards, brouettes et autres chariots; ceux liés à la construction de l'habitat: charpente, maçonnerie; ceux liés à la pratique du feu, à la cuisine, etc., leur apprentissage et leur transmission donc, s'est faite sans que l'écrit n'ait été jugé nécessaire. Les modalités de sauvegarde, de protection et d'accumulation mises au 
point dans le cadre du régime de la pratique ${ }^{18}$, et qui le constituent, se sont avérées d'une redoutable efficacité, non seulement pour maintenir le capital de connaissance technique, mais aussi pour l'accroître. Les groupes humains n'eurent de cesse, en effet, de se déplacer et de s'adapter aux environnements nouveaux ou tout simplement changeants. Les énoncés techniques n'ont pas été spontanément des écrits. La forme écrite des énoncés techniques est arrivée tardivement dans l'histoire des sociétés humaines, et la première quesation à poser historiquement est celle du pourquoi.

Pourquoi coucher la technique par écrit, alors que les modalités d'apprentissage, de mémorisation et de transmission en vigueur dans le cadre du régime de la pratique fonctionnaient parfaitement ? Observons que dans l'environnement technique méditerranéen, les premiers écrits techniques proprement dit, j'entends des textes portant exclusivement sur la description et la mise en œuvre de compétences et de savoir-faire, sont écrits durant la période impériale romaine, et qu'ils se sont intéressés à des compilations architecturales et technoadministratives, pas à des chaînes opératoires. Observons que déjà le monde grec distinguait entre la "technè », l'expertise technique du pilote ou de l'architecte, vantée par Platon, et le "banausos ", l'artisan, placé du côté des choses viles dont l'activité, selon le même Platon, ne pouvait convenir aux citoyens ${ }^{19}$. Observons que l'époque était celle d'un moment fort d'échange commercial, d'unité linguistique des élites autour du grec et du latin, et de normalisation technique, les découvertes archéologiques nous le font comprendre chaque jour un peu plus. Observons enfin, à titre d'exemple, le superbe De Aquis Urbis Romae. Moins connu que le De architectura de Vitruve ${ }^{20}$, il fut rédigé par Frontin $^{21}$, qui exerça en tant que «curator » des eaux de la ville de Rome. La réussite de son ouvrage sur les eaux tient à sa qualité technique et juridique, dans un monde où d'une part, la gestion des eaux étaient essentielle au développement des villes, Rome au premier chef, où d'autre part, toute ville était pen- sée, construite, en prenant l'Urbs, Rome, comme modèle princeps ${ }^{22}$. Ce que je veux dire, c'est que la fortune de ces premiers écrits techniques, qui se traduit entre autres par leur transmission jusqu'à nous, est liée à un substrat culturel dominé par un modèle et ses normes. Il y a donc un public et une raison, la propagation d'un modèle urbain ${ }^{23}$. Nul besoin, en regard d'écrire un traité sur la fabrication des tegulae, par exemple, voire même un simple traité de la forge ${ }^{24}$. L'archéologie atteste de la présence généralisée de ce type de compétences sur tout l'Empire romain, comme elle atteste de la circulation marchande des semi-produits à côté des produits finis ${ }^{25}$... La mise en écrit n'a concerné ni les savoir-faire, ni les métiers, mais très exactement ce que Platon valorisait, l'art de " placer en ordre ses matériaux et forcer chacun à s'ajuster et à s'harmoniser au voisin, jusqu'à composer un tout bien arrangé et bien ordonné ${ }^{26}$. J'ai pris volontairement l'exemple romain pour souligner qu'en matière d'énoncés techniques, passer de l'oralité à l'écriture renvoie à une finalité autre que la transmission du geste technique, de la chaîne opératoire ou du procédé, et cela, très probablement, indépendamment du temps, du lieu et de l'époque. Il y a là bien plus qu'un évènement dans une chronologie. Il $\mathrm{y}$ a un "moment " historique, manière d'évènement interne dans une structure sociétale donnée. Moment de passage, moment de compréhension neuve de l'art d'écrire, moment de recomposition des ordonnancements productifs, en l'occurrence. Mais la principale leçon, pour ce qui nous occupe ici, c'est que l'intentionnalité structure l'écrit technique, comme elle le fait de tout écrit.

Ce qui caractérise ces énoncés, à nos yeux, c'est leur efficacité, l'aide à l'action qu'ils procurent. Les écrits sont là pour nous montrer comment faire, pour nous guider le long d'une chaîne opératoire, dans la prise en main d'un nouvel objet matériel, ou immatériel, ou pour améliorer notre pratique. Tous, nous les utilisons. Il y a ceux du quotidien: la grammaire Ikea, et sa sémiologie caractéristique, est connue de tous désormais. Et chaque jour, ou 
presque, nous lisons sur leurs emballages, les conseils techniques pour réaliser une purée, un gâteau, une crème, un pain en machine, etc. Il y a ceux que nous fréquentons pour perfectionner une pratique : les revues spécialisées dans le bricolage, en informatique, en jardinage. Il y a ceux que nous lisons pour nous former ou nous cultiver, à quoi appartiennent les séries très à la mode des « écrits pour les nuls », qui sont d'excellents manuels de réduction en art, version contemporaine. Leur univocité nous semble naturelle et c'est à peine si nous notons qu'ils s'accompagnent de pictogrammes ou de dessins explicatifs, voire de lavis, dans le cas des magazines. La finalité l'emporte sur les moyens scripturaires utilisés. Singulièrement réduite, cette approche spontanée de l'énoncé technique est le fruit de la culture dans laquelle nous baignons, j'entends ici, culture globale plutôt que sous-cultures sociales, professionnelles, générationnelles ou sexuées. Précisément. La culture dominante en termes de relation à la technique, le fondement dans lequel cette relation s'inscrit, prend forme et grandit, relève du régime de la technique; en regard, le régime de la technologie dicte la conception des objets et des procédés qui nous sont proposés.

C'est dire qu'à notre insu, nous considérons naturel, le fondement et le signe même de la technicité la précision des dénominations, des écrits et de l'iconographie qui l'accompagne, la précision des normes et des nomenclatures. Du fait de notre culture, nous assimilons de manière intangible, la précision à l'univocité et l'univocité au chiffre, au quantitatif. Et nous faisons de l'ensemble la condition sine qua non de l'efficacité. Non sans raison, il est vrai. Un geste précis, «calculé » est toujours plus efficace qu'un geste incertain. La précision facilite la nomenclature, donc la rationalité, donc une plus grande efficacité, y compris dans la transmission. En d'autres termes, le régime de la technique, tel qu'il s'est développé à partir du XVIe siècle en $\mathrm{Eu}-$ rope, a conféré à l'écrit technique des qualités qui relevaient jusque-là du geste dans la chaîne opératoire. La caractéristique du geste est devenue celle de l'énoncé technique : précis, univoque, fondé en raison. Un pas supplémentaire a été franchi avec le déploiement du régime de la technologie, dans lequel nous vivons aujourd'hui. Ce régime spécifique, parce qu'il travaille non pas à mettre l'objet en adéquation mais à concevoir simultanément l'usage et l'objet, en normant conjointement l'usage, l'objet, ses composants, et l'usager, utilise abondamment les habitudes qu'a forgées en nous le régime de la technique. Cela l'autorise à démultiplier l'univocité à son avantage. Fondement du marché global du meuble, la notice de montage Ikea est à peu près devenu un langage planétaire, tout comme le maniement des smartphones, et l'usage des « apps », fondement de l'économie numérique globale.

On comprend du coup que l'énoncé technique fasse rarement l'objet de recherches historiques, qu'il soit rarement considéré dans son historicité. C'est exactement le point aveugle de notre regard sur la technique. L'attitude courante à son égard est informative. Il n'est pas spontanément pensé comme un énoncé situé historiquement. Il est utilisé pour ce qu'il apprend des techniques dont il parle ; il est requis, mobilisé pour l'information qu'il apporte, pour les renseignements qu'il apporte sur les techniques considérées. Cela est surtout vrai des techniques qui aujourd'hui n'existent plus ou celles dont on essaye de comprendre le fonctionnement, de recréer le savoir-faire. Les Manuels Roret demeurent très pratiqués pour les connaissances qu'ils apportent de procédés techniques anciens. Les artisans d'art, les archéologues, les restaurateurs ou les historiens des techniques qui cherchent à améliorer les connaissances des matériaux et des procédés au moyen de l'expérimentation, y recourent spontanément. Les mêmes recourent à l'Encyclopédie de Diderot et d'Alembert, moins fréquemment, à l'Encyclopédie méthodique, et à toute la littérature technique qui s'est développée en Europe depuis l'invention de l'imprimé. C'est ce que j'appelle l'attitude prédatrice: lorsque nous voulons comprendre, ou tout simplement mieux connaître une technique historique, une technique développée 
dans une culture historiquement révolue, nous nous tournons vers la littérature historiquement disponible, et nous l'utilisons à peu près comme s'il s'agissait d'un manuel, et comme si ce manuel avait été rédigé à notre intention, pour notre formation. L'énoncé technique est pris pour ce qu'il paraît, un énoncé destiné à faire connaître quelque chose de la technique, à rendre possible son apprentissage et sa pratique. Il est appréhendé comme totalement neutre, pour ne pas dire transparent, et totalement explicite. Il est pensé en dehors de l'historicité, étranger à elle. Ou du moins, il n'aurait d'autre historicité que celle de l'objet ou du procédé auquel il est dédié, et dont il décrit le fonctionnement. Son historicité serait incluse, enchâssée dans l'historicité de l'objet, du procédé, du produit décrit, présenté, expliqué, et en serait tributaire.

Or, cela n'est pas. Cela n'est pas, et pour plusieurs raisons. La première relève de la logique pure. Postuler que seul l'objet technique est porteur d'histoire, revient à postuler l'universalité de l'énoncé technique. Un forgeron chinois aura certainement les mêmes gestes qu'un forgeron africain, ou européen. Et peut-être même leurs ateliers seront-ils organisés de manière assez proche, assez analogue, à défaut d'être absolument identique. De même, un pêcheur à la ligne ou au filet. De même, un potier. Cela s'explique par ce qu'André LeroiGourhan a qualifié de «tendance technique » ${ }^{27}$. Ce qui est certain, par contre, c'est que l'inscription du fait technique «forger », ou «pêcher » ou « filer » ou «tisser» dans la topique conceptuelle, l'inscription du geste, ou si l'on veut de l'acte, dans la dynamique image/mot diffère totalement. Le forgeron ne pense, ni ne conçoit son atelier de la même manière selon qu'il est Chinois, Africain, ou de quelque endroit d'Europe. La représentation mentale qu'il s'en fait, la carte mentale qu'il en dresse, qu'il établit pour évoluer « instinctivement » dans son atelier, diffère, parce que les milieux culturels sont différents. Ce qui est universel, c'est qu'il verbalise sa technicité et l'énonce. C'est la spécificité de l'acte technique humain. Mais l'expression de cette verbalisation diffère selon les civilisations et leurs cultures, selon les manières qu'ont les multiples sociétés humaines de dénommer, de dénombrer, d'exprimer le temps et l'espace, de verbaliser leur environnement. Et au sein d'une civilisation, le changement est susceptible d'intervenir selon les époques. La conceptualisation du fait technique, sa perception varie en effet, selon les époques, en fonction de la disponibilité des outils. Il n'y a pas d'universalité de la littérature technique, ni dans ses formes, ni dans son expression ${ }^{28}$. Tout énoncé technique est situé culturellement ${ }^{29}$.

La seconde raison relève de cette historicité précisément. Dans toute civilisation, dans toute culture, l'écrit est second. Et toute civilisation, toute culture qui s'est dotée d'une relation structurée à l'écrit, a nécessairement élaboré cette relation. Elle est nécessairement passée par diverses phases d'apprentissage, d'acclimatation et de maîtrise. L'histoire a capté quelques-unes de ces réorganisations, la plupart, pour ne pas dire à peu près toute, provoquées par une modification interne au régime de l'écrit. Ainsi, le passage du rouleau au support relié a eu pour effet une modification des habitudes de lecture. Ainsi encore : on sait combien la Renaissance européenne a été l'une de ces phases de remaniement des régimes de savoir, autour d'une nouveau type d'écrit, l'imprimé. Une autre réorganisation s'est faite, il y a peu avec le déploiement de l'économie numérique, et sans doute n'est-elle pas achevée. Elle débouche sur une approche très fragmentée, très segmentée des savoirs, leur changement de statut de la connaissance vers l'information, un régime de la preuve qui privilégie l'accumulation, et un retour en force de l'image dans le raisonnement. Ce que l'on connaît moins, parce que cela a été très peu étudié, ce sont les modalités de passage, locale et générale, d'une acquisition des savoirs et des connaissances structurée autour de l'oralité à une structuration par l'écrit. Et ce qui a été plus négligé encore, c'est la manière dont les diverses sociétés et cultures ont élaboré une relation écrite à la technique. Or les textes témoi- 
gnent nécessairement de cette historicité de la relation ; ils en portent nécessairement la marque. Cette marque, nous l'enregistrons, mais presqu'exclusivement dans le sens d'un perfectionnement et d'une maîtrise plus assurée de l'efficacité de ce type de production écrite. Il n'est pas encore fréquent d'analyser la différence entre les écrits techniques, comme l'indice d'une approche autre, différenciée à l'écriture de la technique, comme une possible différence de conception, comme la trace d'un changement de régime dans l'élaboration des topiques de pensée liés au fait technique. C'est à peine si la question est posée de l'établissement et de la transmission de ces textes.

La troisième raison tient à la spécificité, pour ne pas dire la particularité, de la relation explicite/implicite dans le cas du texte technique écrit, qu'il soit publié ou manuscrit. Implicite : c'est sans conteste le plan de référence le plus difficile à appréhender et le plus fantasmé aussi. Sa traduction historique la plus fréquente est venue du monde anglo-saxon, avec l'expression "tacit knowledge ", dont la traduction en français se fait souvent de manière littérale, «savoirs tacites ». Or, cette traduction littérale, quoique devenue canonique, est inappropriée, les deux termes ne décrivant pas en français la même réalité. Un savoir «tacite» est, par définition, un savoir que l'on tait. Or taire quelque chose suppose deux attitudes complémentaires, savoir que l'on sait d'une part, choisir de ne pas le dire d'autre part. Il est certain que taire un savoir le fait basculer dans l'implicite. Mais pour qui ? La catégorie de l'implicite, si elle renvoie par définition à ce qui n'est pas dit, à ce qui n'est pas explicitement exprimé, va bien au-delà de ce que l'on tait. Elle exprime ce qui n'a pas besoin d'être dit (c'est le sens même du terme implicite) et ce qui est compris inconsciemment sans être dit. Le tacite renvoie au choix de dire ou de ne pas dire; l'implicite renvoie à ce qu'il est inutile de dire, parce que déjà présent dans les plis du langage, dans ce qu'on sous-entend. Et sous-entendre suppose d'organiser le langage de manière à ce que chacun capte le sous-entendu, l'entende en somme sans qu'il soit besoin de le dire. Taire quelque chose, créer une entente tacite, relève de l'économie personnelle ou sociale, et ne suppose pas une compréhension générale. C'est même souvent le contraire. Sous-entendre quelque chose, le placer dans l'implicite donc, relève de l'économie du langage, et suppose, pour sa compréhension, de se positionner dans le référent culturel, culture globale d'une société toute classes confondues, culture spécifique d'un groupe social ou professionnel. L'implicite est rarement particulier. Ce qu'il importe de comprendre à ce stade, est que tout langage humain, comporte une part importante d'implicite, et que cet implicite diffère en fonction du vécu des sociétés et de leurs habitudes culturelles. C'est même cela qui pour une grande part justifie le métier d'historien. Son travail consiste, pour une part non négligeable à comprendre et restituer l'implicite des textes anciens. A l'époque de leur écriture, on pouvait les comprendre à demi-mots. Les changements sociaux et culturels les rendent difficilement audibles, voire plus du tout.

La difficulté, en Histoire des Techniques, est de détecter et de comprendre, au-delà de la neutralité apparente d'expression, ce qui relève de l'implicite. Un énoncé sera communément qualifié de neutre lorsqu'il est indemne de polémique et d'idéologie, lorsqu'il ne contient et n'exprime aucune valeur autre que celle se rapportant strictement à son objet, lorsqu'il est vierge de signification complémentaire et qu'il ne charrie avec lui aucun métadiscours. Ainsi, l'énoncé « prenez trente grammes de beurre, un œuf, cinquante grammes de farine, trente grammes de sucre, mettez-les dans un récipient et battez-les au fouet jusqu'à obtenir une pâte homogène " sera de prime abord considéré comme un énoncé parfaitement neutre : il se rapporte strictement à son objet, ne contient en apparence aucun métadiscours, ne véhicule aucune valeur sociétale, et se réfère strictement à son objet : une recette de cuisine. Sauf qu'il ne peut être compris que dans le contexte qui l'a engendré, celui d'un savoir-faire 
cuisinier habitué à la manipulation du lait et des œufs, connaissant le contenu des termes « beurre » et « sucre », habitué de surcroît à user du système métrique, donc à peser en grammes, et disposant de moyens techniques autorisant cette mesure... Il prendra donc difficilement sens dans une cuisine japonaise, et encore moins dans une maison Jivaro en Amazonie... L'énoncé est neutre, c'est certain, mais sous-tendu par une série d'implicites qui brouille sa compréhension en dehors du contexte culturel natif. En fait, les trois questions : neutralité, intentionnalité, degré et nature de l'implicite doivent être croisées en prenant la précaution de ne placer aucune nécessairement en lien de causalité par rapport aux deux autres, et aucune, en relation de causalité, disons d'automaticité causale, avec la situation sociale des acteurs.

Autrement dit, à quoi pense le rédacteur d'un texte technique ? Qu'exprimera-t-il explicitement et pourquoi ? Quel sera son sentier de dépendance ? Autrement dit, qu'est-ce que l'économie de l'énonciation lui imposera ou le conduira à placer dans l'implicite, en s'appuyant sur le capital de connaissance commun ? La finalité du texte, plus exactement son intentionnalité va certainement guider la stratégie d'écriture du rédacteur, voire de l'éditeur, et décider de son positionnement par rapport aux cultures ambiantes, culture générale et cultures spécifiques, si le public visé est socialement déterminé. Il est naturel en regard de considérer que cet auteur, ce rédacteur, déploiera en conséquence les moyens techniques d'écriture et de mise en valeur du texte qu'il considère nécessaire, indispensable à la réussite de son résultat. Sans toutefois que cela rejaillisse automatiquement sur la relation auteur-lecteur: les choix rédactionnels et de mise en forme de l'auteur peuvent trouver leur public, mais pas nécessairement. Si les choix sont adéquats, la publication, manuscrite ou imprimée, emportera l'adhésion ; elle sera signifiante socialement. S'ils sont erronés, trop innovants, trop en décalage ou trop rétrogrades, elle manquera son objet.

C'est cette intrication entre culture, volonté édi- toriale et impact sociétal souhaité et obtenu, qui doit déterminer l'analyse historique, et non le désir de l'historien de comprendre le fonctionnement de l'objet, du procédé ou du processus qu'il a décidé d'analyser. En d'autres termes, une analyse historique, pour être fructueuse doit se focaliser sur l'intentionnalité du texte et non sur celle de l'historien. A la grille d'analyse habituelle : de quoi ça parle, comment ça en parle, dans ce cadre, qu'est-ce qui est absent, qu'est-ce qui n'est pas dit, enfin qu'est-ce qui est inusité, inhabituel dans la manière dont s'est dit, qu'est-ce qui me choque, grille que tout jeune chercheur, tout historien débutant, apprend à poser et à maitriser, se rajoutent une batterie de questions complémentaires. Viendront en premier les questions se rapportant à l'auteur, aux buts qu'il s'est fixé : transmission par la pédagogie d'un objet courant, transmission par la pédagogie d'un objet innovant; découverte, mise à disposition d'un objet, ou d'un savoir-faire innovant; présentation d'un usage ou d'une rhétorique innovante à propos d'un objet connu en soi. Il sera nécessaire en complément d'interroger la place habituelle de l'écrit dans la culture du temps, et dans ce cadre, envisager la question spécifique de l'écrit technique : est-il habituel ou au contraire, est-il exceptionnel; l'écrit est-il un moyen habituel de pédagogie technique, de pédagogie générale. Il sera indispensable enfin d'évaluer le positionnement sociétal du ou des rédacteurs, de manière à évaluer correctement cette autre aspect de l'intentionnalité : l'écrit est-il élaboré à titre de valorisation personnelle ou collective, voire de marketing culturel? A l'inverse, évite-t-il soigneusement cet aspect ? Quel est la cible, le public visé, prince ou grands, élite, communauté professionnelle, grand public, amateurs éclairés ? Souvent la recherche se focalise sur l'intentionnalité sociétale d'un écrit technique. Et dans ce cas, souvent, la recherche postule une action positive, entendons une recherche de positionnement, une volonté de valorisation du travail effectué, ou de la personne concernée. Or, il n'est pas certain à l'échelle de 
l'histoire, et de nos jours encore, que l'écrit technique soit pour un auteur le moyen le plus direct de valorisation sociale pour un auteur. En tout état de cause, il est difficile de postuler que c'est là sa fonction première. Si fonction sociétale il y a, et cette fonction existe, elle est à postuler, en premier lieu, par rapport à son domaine, le monde de la technique. On posera alors les questions suivantes : à quoi sert-il d'écrire de la technique ? Quelles techniques sont écrites, et quelles sont négligées ? Quel est le rôle de l'écrit technique dans la communauté professionnelle impliquée ? Où en est cette communauté professionnelle dans la constitution de son habitus technique ${ }^{30}$ ? Cherche-t-elle à doubler cet habitus technique d'un habitus social ?

Il est deux présupposés à ne pas négliger, pour qui s'intéresse à l'écrit technique, pour l'utiliser et/ou l'étudier : 1) si l'objet, le procédé ou le processus est proposé à la lecture d'un public quelconque, c'est qu'il détonne ou étonne, qu'il rompt avec les habitudes d'une manière ou d'une autre, qu'il requiert un apprentissage, une " mise à niveau culturelle »; si un auteur, individuel ou collectif, décide de consacrer un écrit à un objet, un procédé ou un processus technique, c'est d'une part que cet objet, ce procédé, ce processus interroge et/ou pose problème techniquement, économiquement, commercialement, ou culturellement, ce qui rend nécessaire des formes spécifiques de mise en familiarité, d'autre part, qu'il considère que l'écrit technique est l'outil idoine pour faire face à la situation ; 2) sachant toutefois que personne, dans quelque lieu et dans quelque culture que ce soit, y compris de nos jours, n'apprend à pratiquer, à faire fonctionner un objet en lisant un écrit technique, fût-il un mode d'emploi. La seconde proposition paraît d'emblée en contradiction, en porte-à-faux avec la première, en porte-à-faux également avec les habitudes des uns et des autres, surtout s'agissant de mode d'emploi. Pour le comprendre, revenons vers la notice de montage Ikea. Son intentionnalité est patente : un écrit pensé pour faciliter une pratique, le montage de meubles, et faire qu'il soit opérant à l'échelle de la planète. Tous, nous avons bénéficié de l'efficacité de cette mise en forme qui repose sur deux éléments structurants : la détermination d'une chaîne opératoire du montage rigoureusement déterminée (élément 1), sa mise en image (élément 2). Tel est l'explicite. Tout le reste, l'implicite, est en fait à ce point naturel que nous n'y pensons pas. Nous ne pensons pas, dans le suivi de la chaîne opératoire, à la manière de dénombrer. Elle nous paraît naturelle parce qu'elle repose sur la méthode occidentale, est conforme à nos habitudes : c'est le premier implicite. Or, une notice de montage pensée en prenant pour base la méthode chinoise de dénombrement procéderait autrement. J'ai pu vérifier, par mon expérience personnelle, qu'elle était totalement incompréhensible aux yeux d'un européen $^{31}$. Nous ne pensons pas non plus, à la manière de tenir un marteau, un tournevis, etc. Nous n'y pensons pas parce que nous savons les utiliser, nous avons appris à le faire auparavant, en d'autres lieux, en d'autres endroits. C'est le second implicite. Enfin, nous trouvons naturel de ne pas utiliser d'autres outils : perceuses, forêts, etc. Nous le trouvons naturel car le montage du meuble que nous avons acheté a été prévu ainsi : c'est le troisième implicite. Bref, nous ne sommes pas devant un mode d'emploi, mais devant une notice de montage qui a été réfléchi, conçue en prenant en compte, le savoirfaire basique de la relation au bricolage : visser essentiellement, et frapper au marteau. Or ce savoirfaire basique : visser, utiliser un marteau, personne ne l'a appris dans un livre, ou à partir d'un mode d'emploi. Il relève exclusivement de la pratique, et son perfectionnement de l'expérience. C'est même la difficulté inhérente à tout mode d'emploi. Il est naturel à tous qu'une pratique s'acquiert par la pratique... Il n'est pas naturel d'initier une pratique en lisant un document. L'approche spontanée, que nous expérimentons tous, est de tenter de faire fonctionner l'engin que nous venons d'acheter. Et c'est lorsque nous échouons que nous allons voir le mode d'emploi. Il n'y a pas contradiction entre la nécessité d'intégrer un savoir-faire par la pratique, 
et de sa familiariser avec lui par l'écrit. Mais bien une tension intéressante à analyser, aux deux plans de l'anthropologie et de l'histoire, à condition de comprendre quel(s) régime(s) de pensée opératoire imprègnent, ou dominent, la culture technique ambiante, technique ou technologique, en l'occurrence.

L'écrit technique n'est pas une transcription brute, une simple mise à plat de l'acte technique. Tout bien réfléchi d'ailleurs, la décision de mettre en écrit l'acte technique, est contre-nature. Rien de banal dans cette opération, qui consiste fondamentalement en une intellectuation de l'action, un renforcement de la mise en mots, et l'affaiblissement consécutif de la mise en actes. Rédiger, c'est réduire à deux dimensions un objet normalement appréhendé en trois, voire en quatre dimensions, si l'on inclue la facette du « sensible », bien souvent constitutive du geste technique et/ou du procédé concerné, et, en tout état de cause, absolument nécessaire à sa mise en acte. Le couple mise en mots/mise en image sera donc fortement sollicité, et travaillé pour déboucher sur une efficacité, qui, pour une part, sera appréciée, elle aussi, à l'aune de l'habitus culturel ambiant. Mais efficacité de quoi ? Le résultat recherché peut-être la reproduction du geste, du procédé ou de l'objet. Mais est-ce possible? Est-il réellement possible, à partir du seul couple «lecteur/rédaction » de mettre en œuvre l'apprentissage par essais/erreurs/correction dont on sait qu'il est nécessaire à la maîtrise de tout geste, de toute action reproductible? Le résultat est-il la connaissance du geste, du procédé ou de l'objet, ou, à tout le moins, une mise en familiarité qui constitue la première étape du savoir technique, l'introduction au savoir-faire? L'écrit technique, dans cette hypothèse, aurait pour fonction première d'initier le lecteur, de l'introduire dans les rudiments intellectuels de la pratique, d'opérer une mise en image et une mise en mots, qui favoriseront ensuite éventuellement, si le lecteur est motivé, intéressé, et doué, la mise en acte. L'écrit facilitateur de la pratique? Pourquoi pas, dans les sociétés où l'intellectuation l'a définitivement emporté sur l'appréhension sensible. Mais, y compris dans ce cas, l'écriture de la technique est une construction, elle implique des choix, dans la description, la mise en forme, qui à la fois s'inscriront dans la culture ambiante, dans l'écosystème intellectuel, qui useront des outils disponibles, aussi bien pour la mise en mots que pour la mise en images. Je reviendrai sur ce dernier point. Quoiqu'il en soit, cet usage « pédagogique » de l'écriture technique n'est pas le plus fréquent. Il suppose en effet, pour sa mise en œuvre, que l'histoire culturelle et professionnelle de la société concernée ait placé l'apprentissage technique principalement du côté de l'écrit, ce qui, y compris de nos jours, dans les sociétés les plus intellectualisées, n'existe pas encore. Même en France, les manuels de «techno » viennent en renfort de la pratique, et non à sa place...

De fait, le but d'un écrit technique est rarement d'initier un fonctionnement. Répétons-le : personne n'a appris à forger, à tisser, à produire du verre ou à faire tourner un tour de potier, en lisant un manuel, personne n'a appris à nager, à faire du vélo, à pratiquer un sport ni même à photographier, en lisant un manuel. L'intention de G. Agricola durant les trente années d'élaboration de son grand oeuvre sur le métal, le De re metallica, n'a pas été de rédiger un traité d'exploitation minière et de production de métal. Les maîtres mineurs et fondeurs n'en n'avaient pas besoin. Agricola s'en explique d'ailleurs dans sa préface introductive. Son but n'est pas de dévoiler des tours de main; il est de donner aux administrateurs et entrepreneurs une approche raisonnée, scientifique, de la totalité de la chaîne opératoire minéro-métallurgique, la somme la plus complète possible, en intégrant les variantes régionales, et les spécificités de production de chaque métal, de manière à leur donner les moyens de comprendre, d'interpréter et donc de gérer ce qui était alors la première industrie européenne, à côté de l'industrie textile, et la plus importante quant à l'investissement technique et financier. Le De re metallica ne vise pas le public des praticiens, il vise celui des lisant-écrivant, le public des administra- 
teurs et des gestionnaires ${ }^{32}$. Et la démonstration à leur égard est double : oui, il est possible d'écrire sur la pratique, oui, écrire sur la pratique, la décrire donc, du moins dans ses grandes lignes, si ce n'est dans le détail des procédés, aide à mieux gérer, facilite l'introduction dans la production, d'une temporalité nouvelle : la longue durée, tandis que le praticien, par nécessité, déploie son activité dans la courte durée du procédé qu'il maîtrise. Grammairien, médecin, échevin, G. Agricola n'avait pas besoin du De re metallica pour se positionner dans la société et asseoir sa réputation, même si Érasme lui-même salua son projet. Il était un homme respecté et apprécié, posé socialement ${ }^{33}$. De même, C. A. Schlutter, lorsqu'il écrit son traité des fontes, destiné à devenir célèbre dans le monde des savants métallurgistes, ne cherchait pas à apprendre aux praticiens à produire du métal ${ }^{34}$. Son but était scientifique, ou plus exactement technologique, au sens où l'entendaient alors les philosophes allemands dans leur enseignement universitaire, d'élaboration de bibliothèques de connaissances ${ }^{35}$, et plus précisément, d'élaborer autour de la production de métal, un corpus de connaissances, relevant non de la technicité, mais de la connaissance intellectuelle, de la théorie sur la technicité. Le public visé, là encore, était les administrateurs, savants. Avec la volonté de renforcer la coactivité avec les praticiens, soit pour l'administration, soit pour l'expérimentation et l'innovation, trois pratiques en plein essor depuis le milieu du XVII ${ }^{\text {siècle }}{ }^{36}$. L'intentionnalité des Voyages métallurgiques de Gabriel Jars, rédigés entre 1751 et 1769 est proche ${ }^{37}$. L'ingénieur distinguait parfaitement les deux domaines, de la pratique et de la connaissance technique ou technologique. Jars, on le sait, n'hésitait pas à montrer aux praticiens comment se servir d'un nouvel objet ${ }^{38}$. Et, à l'intention des entrepreneurs, des administrateurs et des savants et en leur compagnie, il travaillait par l'écrit, à constituer une métallurgie scientifique, qui fut qualifiée alors de "sidérurgie ", pour la distinguer de la métallurgie courante. Autrement dit, le but des écrits sur la mine et le métal entre XVI et
XVIIIe siècle fut bien de fonder et de fixer des modalités neuves de connaissances et pour ce faire, d'utiliser la puissance conjuguée de l'écrit et de l’imprimé.

Continuons. Et passons du XVIII ${ }^{e}$ au XIX ${ }^{\mathrm{e}}$ siècle, de l'époque de l'industrie «industrieuse » à celle de l'industrie "industrielle », du monde industrieux au monde industriel. Quoi de plus neutre en apparence qu'un article des Annales des Mines, quoi de plus inutile en apparence, que de le confronter à ses équivalents, que d'établir une comparaison avec les Comptes rendus de la Société de l'Industrie Minérale en France ou le Journal de la Société des Ingénieurs civils. Tous sont des écrits d'ingénieurs. Tous paraissent gravés dans le marbre de la neutralité, et marqués du sceau de l'objectivité. Le ton est impersonnel, solidement établies, les normes rhétoriques usent des règles de la science expérimentale. Les sujets sont proches, pour ne pas dire identiques. Des sujets analysés objectivement, des photographies froides de procédés, placées là, à titre d'enseignement, de mise à disposition, de compréhension, voire d'utilisation par le lecteur. Des sujets techniques : une usine, une mine, un procédé ; un objectif : donner à voir; un outil rhétorique : la démonstration scientifique; une même origine socio-professionnelles : des ingénieurs. Tout plaide pour l'unicité, et fait de cette unicité, une univocité aisément traduite par le lecteur en neutralité. S’il cède à ces apparences, l'historien sera le dupé de l'histoire. Car il négligera la question centrale : ces textes techniques du XIX ${ }^{\mathrm{e}}$ siècle, quel est leur objectif?

C'est le propre de l'historien de s'intéresser au «back-office », d'interroger les lieux et groupes qui fixent les lignes éditoriales. Or, qui dit ligne éditoriale, dit politique et choix éditoriaux. Pourquoi les Annales des Mines mettent-elles en avant l'industrie du zinc dans les années 1840, et pourquoi est-il tellement question de l'acier et de la sidérurgie dans les années 1870 dans les livraisons des Comptes Rendus de la Société de l'Industrie Minérale? Et pourquoi pas l'inverse? Pourquoi tant 
d'articles consacrés au brassage mécanique des métaux dans les Annales des Mines dans les années 1850 ? Pourquoi tant de place donnée à la sidérurgie au bois ? Et pourquoi si peu consacrés à la production de coke ? Les minutes des comités de rédaction, lorsqu'elles sont disponibles, donnent à voir, derrière la façade rhétorique de l'imprimé final, la force des tensions, des oppositions allant jusqu'à la fracture, des inflexions exprimant la reprise en main, tous faits qui traduisent les luttes d'influence, quand elles ne signent pas, d'un comité de rédaction à l'autre, l'opposition entre cultures techniques ${ }^{39}$. La formalisation imposée par l'imprimé accroît la force démonstrative de l'énoncé ; elle est aussi outil de constitution des habitus techniques, de renforcement des identités techniques, et de légitimation sociale. Les politiques éditoriales expriment des positionnements socio-techniques, que l'objectivité rhétorique masque et conforte à la fois. Tous partagent l'objectivité rhétorique, posé là en manteau commun. Cela signe une époque où chefs d'entreprise et ingénieurs s'accordaient pour réduire l'incertitude, accroître les profits, développer l'innovation en faisant de l'industrie un objet de science, en construisant autour de l'industrie, une culture, un ordre scientifique spécifique. A partir de quoi, chacun des comités de rédaction se positionne en fonction de ses intérêts et questionnement spécifique : intérêts économiques et/ou politiques, questionnements techniques.

L'étude de ces textes techniques dévoile la place occupée par chacun dans un procès de production marqué par l'expansion permanente. Les ingénieurs du corps des Mines, seuls à pouvoir disposer officiellement du titre, font des Annales, l'expression d'une politique techno-économique, et veillent à donner le ton, voire à anticiper sur l'évolution. Leur position est fondamentalement doctrinale. Les ingénieurs de la Société de l'Industrie Minérale ont, eux, les mains dans le cambouis. Ils ont en charge, particulièrement les ingénieurs métallurgistes, le développement d'un secteur phare de l'expansion industrielle, le secteur sidérurgique. Leur position est fondamentalement praticienne, d'échanges de savoir-faire, de constitution d'une base de connaissance, qui les aide à faire face au passage de la sidérurgie au coke, puis à la crise technique induite par la mise au point du procédé Bessemer. Leurs préoccupations moins ciblées nécessairement, témoignent d'un monde en forte expansion, quoiqu'il ne soit pas encore marqué au fer de la concurrence exacerbée. L'objet commun est la construction des infrastructures, la détermination des espaces et outils de circulation économique et technique. Une expansion dont il faut observer, par ailleurs, qu'elle s'opéra sans accroissement notable de l'entropie économique ou technique : c'est là une caractéristique de l'écologie du système industriel au XIX ${ }^{\mathrm{e}}$ siècle, qui en fait une différence fondamentale avec l'écologie de ce même système au $\mathrm{XX}^{\mathrm{e}}$ siècle. Cela a facilité la stabilité de la littérature technique; en corollaire, cela renforce à distance, la sensation de neutralité.

En d'autres termes, la neutralité elle-même, l'objectivité apparente de ces textes, leur apparente indifférence au contexte, n'échappe pas à l'historicité, notion à placer ici, dans le cadre défini par H. G. Gadamer, de l'historicité de la compréhension ${ }^{40}$. L'écrit technique a son histoire, dont les grandes lignes, en Europe, définissent l'instauration progressive du régime de la technique entre XII e et XIX ${ }^{\mathrm{e}}$ siècle : début du basculement de la technicité dans le régime de la technique, avec le développement des normes juridico-techniques, en particulier autour de l'économie européenne du textile, entre $\mathrm{XII}^{\mathrm{e}}$ et $\mathrm{XIV}^{\mathrm{e}}$ siècles; élargissement à l'écriture des privilèges, puis, au XVIe, un saut à la fois quantitatif et qualitatif est effectué avec le paradigme de la réduction en art qui structure le langage de l'action jusqu'au XVIII e siècle. Au XVII' siècle, l'écrit technique s'enrichit du croisement avec la philosophie de la connaissance d'une part, en particulier la détermination quasi-grammaticale de la technologie, du développement des sciences expérimentales d'autre part (et réciproquement) : la recherche devient alors, celle d'un «langage universel » de la 
technique, nécessairement écrit, et ainsi codé qu'il transcende les cultures et les époques, parfaitement exprimé par Leibniz, qui avait en commun avec Galilée, d'avoir débuté comme ingénieur.

Puis le XIX siècle, époque glorieuse de l'ingénieur d'industrie, renoue avec le pragmatisme opératoire, avec pour objectif partagé, le progrès technique. La littérature technique se développe alors comme jamais, sous forme de revues, de cours, d'encyclopédies. Voilà pour les grandes lignes de l'évolution de ces écrits, à partir desquelles, il est possible de les contextualiser. Mais, il est, en regard, une autre historicité, celle de l'idée que les divers acteurs et auteurs, se sont faites de son essence, de ses fondements épistémologiques, son ontogenèse en somme. Cette historicité intéresse non la manière dont elle a été élaborée, mais la manière dont elle a été conçue. Concevoir un énoncé neutre et objectif, à cette aune, quoiqu'il ne relève pas de l'obligation juridique, prend allure de victoire de l'esprit, ce pourquoi certainement l'attachement demeure si vif à son égard. L'objectivité a primé. G. Agricola plaide, dans son De re metallica, pour l'universalité des termes techniques et pour une adéquation étroite entre sens et signification ${ }^{41}$. On le sait, déjà, F. Bacon, son aîné avait insisté sur la nécessité de se débarrasser des «idoles», pour aller de l'avant dans l'amélioration et l'accroissement des connaissances.

Cette volonté, nettement exprimée au XVI ${ }^{\mathrm{e}}$ siècle, d'une écriture technique "froide ", universelle, d'une expression normée de manière à procurer une lecture permanente, universelle des textes concernés, de manière, en somme, à leur épargner les contingences de temps et de lieu, s'affirme dans les siècles suivants. Les manifestations en diffèrent toutefois. Neutralité et objectivité, à partir du XVII ${ }^{\mathrm{e}}$ et tout le long du XVIII siècle, prennent une autre couleur. Leur conception adopte un autre contour, qui n'efface pas, loin s'en faut, la conception précédente. Il y a sédimentation. Sous la plume d'un Charles Perrault, d'un Antoine Desgodets au XVII ${ }^{e}$ siècle $^{42}$, il s'agit de révéler les normes, d'analyser leur validité historique et de travailler à leur généralisation, pour gagner en efficacité, en reproductibilité, voire en esthétisme; sous la plume d'un Denis Diderot, d'un Gensanne ${ }^{43}$, d'un Duhamel du Monceau (mais pas d'un Buffon) au XVIII ${ }^{\mathrm{e}}$ siècle, il s'agit par l'expression écrite de mettre en évidence les rationalités propres à tout procédé technique, et jusque-là, masquées, incluses, englobées dans les pratiques artisanales ; la lutte contre la supposée « routine » des gens de métiers devient le maitremot. Extraire la rationalité technique des savoirfaire, la sortir de la mémoire praticienne, l'objectiver donc, pour en accroître la perfectibilité, et, pour accroître le capital technique disponible à l'échelle de la société : cette conception neuve de l'écrit technique débouche sur deux conceptions nouvelles et complémentaires, qui renforcent l'idéal de neutralité, en même temps qu'elles confèrent une légitimité inédite à l'ingénieur de la production, à l'ingénieur d'industrie, l'écriture des brevets d'une part, la conception scientifique de la production industrielle, la scientificité de l'industrie d'autre part. Voilà pourquoi l'historien des techniques doit postuler non une neutralité «en-soi » de l'écrit technique, mais un positionnement historique de sa conception : dans l'écrit que j'analyse ou que je veux utiliser, qu'est-ce qui s'est élaboré, par qui et pour quoi, en termes d'objectivation de l'énoncé technique ? Il doit, bien au-delà du statut social de l'auteur, situer son projet éditorial dans la pensée technique du temps.

C'est dire que tout écrit technique est, comme je l'ai déjà écrit, porteur d'un projet qui n'est pas le fonctionnement en soi de la technique décrite. Le projet d'un écrit technique n'est pas la traduction écrite d'un geste ou d'un procédé, d'une chaîne opératoire. Le déclencheur de l'écrit n'est pas la traduction. Il n'y a pas automaticité du déclencheur. Et puisqu'il n'y a pas automaticité du déclencheur, il y a nécessairement historicité, moment initiateur, intentionnalité. Dans l'écrit technique, l'objet, le procédé, l'acte technique concerné, est inscrit dans un projet intellectuel, et traité de manière à lui cor- 
respondre. Et ce traitement sera nécessairement reflet des outils conceptuels disponibles ou susceptibles de l'être, dans l'hic et nunc du projet d'écriture. Il n'est de neutralité de l'écrit technique que située.

Nous touchons là une difficulté majeure. Car, en regard, les techniques, pratiquées, sont quant à elles, bien réelles et parfaitement objectivées : une cathédrale, une voiture, un navire, un ordinateur, une centrale nucléaire, requièrent pour leur existence l'agence ordonnée d'une somme considérable de compétence, que l'historien et/ou l'archéologue des techniques doit savoir détecter, analyser et comprendre. En bonne théorie, le plan de référence pour mener l'analyse historique des écrits s'y rapportant et déployer correctement la topique conceptuelle, intentionnalité/neutralité/implicite ${ }^{44}$ devrait être la matérialité technique précisément. Or, ce plan de référence fait défaut le plus souvent. Certes, les objets produits sont là, en leur totalité ou sous formes d'artefacts. Mais les agencements matériels et conceptuels qui leur ont donné existence se sont évanouis. En regard, les traces écrites demeurent. Traces matérielles et traces écrites cohabitent dans l'univers culturel et savant, sans se rencontrer, signifiants en mal de signifié, parce qu'au centre, la charnière praticienne, a disparu. Il résulte de cette réalité incontournable, une dispersion des plans de référence. Car, faute de mieux, on s'appuie sur l'écrit pour élaborer une connaissance technique de l'objet ${ }^{45} \ldots$, un écrit qui lui-même requiert pour sa compréhension historique, que soit connu l'ordonnancement technique, chaîne opératoire, complexe technique, qui lui a donné existence, et auquel l'écrit se réfère... jusqu'à un certain point. Dans ce cas, les plans de référence sont comme inversés. On comprend dès lors, la force de la tentation archéométrique, qui tend à devenir ce plan de référence « objectif » qui manque inéluctablement. Sauf que cette objectivité est celle des diagrammes, des images MEB et la spectroscopie RAMAN, pas celle de la technique considérée. Et seule, elle ne permet pas d'établir son histoire, si toutefois établir son « histoire » signifie aussi restituer au plus près l'histoire de sa conception, de ses dénominations, des modalités de transfert, d'insertion, d'apprentissage, la culture technique qu'elle a engendrée ou dans laquelle elle s'est insérée, bref, tout ce qui à côté du " comment » d'un procédé, cherche à répondre au "pourquoi » de son existence et de sa diffusion ${ }^{46}$.

Qu'on me comprenne bien. Il ne s'agit en aucun cas d'aller à l'encontre de l'apport des sciences dures dans la connaissance du passé. Bien au contraire. Joëlle Burnouf a raison lorsqu'elle souligne le rôle épistémologique majeur joué par l'archéométrie dans le renouvellement des études historiques. Et les sciences humaines et sociales spécialisées dans les études historiques de la technique ne pourront faire l'économie du débat qui se mène depuis plusieurs décennies dans le monde anglo-saxon, autour de l'"Historical Archaelogy ${ }^{47}$. Mais, en regard, il n'est pas correct scientifiquement de considérer l'étude historique des techniques, en dehors de leur environnement intellectuel, alors que l'étude de cet environnement est disponible et qu'il existe. $\mathrm{Ni}$ l'archéologie, ni l'archéométrie, ni les sciences environnementales n'ont vocation à remplacer la science historique, $\mathrm{y}$ compris pour l'étude historique des techniques. Mais en regard, il est totalement vain pour l'histoire de continuer à développer ses recherches, en ignorant ou feignant d'ignorer que des méthodes solides et intéressantes d'investigation du passé technique et environnemental, fournissent désormais de nouveaux référentiels et modifient les conditions de réfutabilité. Les archéologues, pas plus que les restaurateurs, ne peuvent négliger l'écrit au motif de son incertitude, ou, position contradictoire et paradoxalement complémentaire, l'utiliser comme référentiel probatoire. Mais en regard, les historiens doivent accepter la nécessité, pour une interprétation pertinente de leurs sources, de se constituer un référentiel qui leur donne simultanément accès à la troisième dimension et à tout ce qui peut rendre concret le geste technique et le procédé. 
Déjà, en son temps, L. Febvre lorsqu'il cherchait à se placer en fondateur de l'histoire des techniques $^{48}$, distinguait ce champ historique de ce qu'il appelait "l'histoire des historiens », parce que le caractère technique de l'objet étudié obligeait à un dialogue permanent avec les praticiens, ingénieurs et scientifiques ${ }^{49}$. Cette réalité ne s'est jamais démentie : les professionnels ont joué un rôle essentiel dans le développement de l'histoire des techniques à l'échelle internationale et en France ${ }^{50}$.

Voilà, en définitive, en quoi le champ " histoire des techniques » se démarque de "l'histoire des historiens ». Il lui faut disposer d'un plan de référence explicitement technicien. Comprendre le fonctionnement d'un four est indispensable pour interpréter correctement les sources sur la métallurgie ou le verre, comprendre les agencements des métiers à tisser est indispensable pour interpréter correctement, ne serait-ce que l'évolution technique, connaître les lignées de roues, et comprendre en quoi elles diffèrent, est indispensable pour retracer l'histoire des transferts techniques et de l'innovation en hydraulique. Rien de tout cela ne s'improvise, ni ne fait partie du bagage culturel dont l'historien dispose " naturellement » du fait de sa culture, à l'instar du politique, du religieux, du social, ou encore de l'économie. Définitivement, la source écrite, manuscrite ou imprimée, est insuffisante à l'historien des techniques, du moins s'il considère que l'objet étudié, la technique, a antériorité sur ce qui l'environne et pour comprendre les interactions réciproques avec ce qui l'environne. Il lui faut, pour interpréter correctement ses sources, prendre à bras-le-corps la technique, appréhender sa matérialité, construire des référentiels adéquats. Ces référentiels, comment les établir, si ce n'est en dialoguant avec les artisans, ingénieurs, architectes, avec les archéologues, les archéomètres et les restaurateurs, en participant à des prospections et des fouilles en archéologie des techniques, en suivant des expérimentations. Cela suppose, il est vrai, coactivité, collaboration et, horresco referens, hybridation...
1 Précision indispensable, tant est forte la tendance actuelle à donner pour historique tout ce qui relève du passé et pour patrimoine tout ce qui est historique.

2 Un patrimoine ne comprend pas que des valeurs positives. Les dettes et déchets que nous léguons à nos descendants, en font partie.

3 En d'autres termes, un lieu de mémoire n'est de facto un lieu d'histoire...

4 Au contraire même, si l'on suit Paul Ricœur : « L'histoire mythique elle-même est au service de cette lutte de la structure contre l'évènement et représente un effort des sociétés pour annuler l'action perturbatrice des facteurs historiques; elle représente une tactique d'annulation de l'historique, d'amortissement de l'évènementiel », RICEUR Paul, " Structure et Herméneutique » (Esprit 1962), Lectures 2. La contrée des philosophes, Paris Seuil, (1992) 1999, p. 366-367. En prolongement de cette affirmation, à propos des indiens Jivaros, TAYLOR Anne-Christine, «L'oubli des morts et la mémoire des meurtres », Terrain, 29, 1997, http://terrain.revues.org/3234, consulté le o9 octobre 2013.

5 DIDEROT Denis, «Explication détaillée du système des connaissances humaines », dans « Prospectus » (octobre 1750), 1751, Euvres complètes, ASSEZAT Jules (éd.), vol. XIII, Paris, Garnier, $1875-77$, p. $129-158$ et 164-165.

6 « Le champ de l'historien est l'histoire sociale et la nature n'existe que comme champ d'expérimentation et d'utilisation par la société, ce qui laisse hors du domaine de la recherche l'essentiel de la réflexion sur la nature », BURNOUF Joëlle, « La nature des médiévistes », Études rurales, vol. 3, n¹67-168, 2003, p. 215-6 ; ou encore : "Nous souhaitons faire valoir ici quelques idées concernant la place des héritages dans l'espace des sociétés. (...) Parce que l'archéologie porte sur des matérialités, et l'archéogéographie sur des formes inscrites dans l'espace géographique, ces deux disciplines peuvent véhiculer un discours sur la dynamique qui ne soit pas exactement le même que celui d'un historien travaillant sur les objets sociaux à partir des documents écrits », BURNOUF Joëlle, CHOUQUET Gérard, «L'archéologie et l'archogéographie : pour comprendre l'espace et ses héritages », dans DEMOULE Jean-Pierre et STIEGLER Bernard, L'avenir du passé, Paris, La Découverte, 2008, p. 93104.

7 Ibidem

8 « Il importe de savoir ce que sont les sources sur lesquelles ces chercheurs s'appuient: ce sont des sources rares, incomplètes, partiales et biaisées. Elles sont rares, c'est-à-dire que plus on remonte dans le temps et moins il y en a (le hasard de la conservation). N'hésitons pas à le dire: elles sont plus rares et plus mal conservées que les sources archéologiques. Elles sont incom- 
plètes et lorsque des fonds ont été conservés ils l'ont souvent été par des copies postérieures, ce qui induit l'établissement d'une 'échelle de sincérité, travail des diplomatistes sur les écritures, les types d'actes et le vocabulaire. Elles sont partiales car elles émanent toujours des pouvoirs et/ou des élites ». BURNOUF, 2003, op. cit.

9 Considérer, comme on le lit encore, que l'Histoire des techniques ne doit pas s'occuper en premier lieu des techniques, c'est considérer que l'histoire économique peut se faire sans l'économie, l'histoire des religions sans la religion, l'histoire culturelle, sans la culture, etc. Bizarrement, cela n'est pas.

${ }_{10}$ Tout historien des techniques doit avoir lu « Ce que peuvent nous apprendre les mots voyageurs ", dans HAUDRICOURT André-Georges, La technologie sciences humaine. Recherches d'Histoire et d'Ethnologie des techniques, Paris, Éd. MSH, 1987, p. 51-56.

${ }^{11}$ FEBVRE Lucien, "Réflexions sur l'histoire des techniques », Annales d'Histoire économique et sociale, $\mathrm{n}^{\circ} 36,1935$, p. 531535 .

12 « Car enfin, les faits... Qu'appelez-vous les faits ? Que mettezvous derrière ce petit mot, « fait » ? Les faits, pensez-vous qu'ils sont donnés à l'histoire comme des réalités substantielles, que le temps a enfoui plus ou moins profondément, et qu'il s'agit simplement de déterrer, de nettoyer, de présenter en belle lumière à vos contemporains ? Ou bien reprenez-vous à votre compte le mot de Berthelot, exaltant la chimie au lendemain de ses premiers triomphes - la chimie, sa chimie, la seule science entre toutes, disait-il orgueilleusement, qui fabrique son objet. En quoi Berthelot se trompait. Car toutes les sciences fabriquent leur objet. », FEBVRE Lucien, "Sur une forme d'histoire qui n'est pas la nôtre. L'histoire historisante ", [Annales (E. S. C.), II, 1947], Combats pour l'Histoire, Paris Armand Colin (1952), 1992, p. 114-118.

13 RICEUR, 1999, op. cit., p. 453-495, MARROU Henri-Irénée, De la connaissance historique, Paris, Seuil, 1954; mais aussi Gabriel Monod, comme le montre LEVY-DUMOULIN Olivier, " Les "Écoles historiques" à l'épreuve de Gabriel Monod. Un historien célébré et méconnu ", Revue historique vol. 4 n 664, 2012, p. 789-801.

14 Lire «L'interprétation en Histoire », RICOEUR Paul, La mémoire, l'histoire, l'oubli, Paris, Seuil, 200o, p. 436-448.

15 En fait «idoles de la caverne» («idola specus»), BACON Francis, Novum Organum (1620), introduction, traduction et notes par Michel Malherbe et Jean-Marie Pousseur, Paris, PUF, 1986, aph. 42.

16 «Idola tribus » et « idola fori », ibidem, aph. 41 et 44.

17 À l'exclusion donc des sources indirectes, juridiques et autres. C'est volontairement que je laisse de côté l'analyse critique de l'iconographie et de son usage en Histoire des techniques.
${ }^{18}$ L'expression désigne l'ordonnancement idéel des savoir-faire dans les sociétés et communautés à dominante orale. GARÇON Anne-Francoise, "Pratique, technique, technologie? ", ArchéoSciences vol. 1, $\mathrm{n}^{\circ}$ 34, 2010, p. 121-126 et GARÇON AnneFrançoise, L'imaginaire et la pensée technique. Une approche historique, $X V I^{e}-X X I^{e}$ siècle, Paris, Classiques Garnier, 2012, p. 149-163.

19 Respectivement dans PLATON, Gorgias ou sur la rhétorique, LIX, 504a et dans les Lois, VIII, 846d.

${ }^{20}$ Ce texte, à la fortune jamais démentie, dispose désormais d'une « app » spécifique sur smartphones et tablettes...

${ }^{21}$ Grand administrateur de l'Empire romain, Sextus Julius Frontinius (30 env.-env. 103) exerça également les fonctions de consul, de général, et de gouverneur de Bretagne. Le service permanent des eaux de la ville de Rome occupait 700 personnes. Excellente introduction au sujet, LEVEAU Philippe, article "Aqueducs », Encyclopaedia Universalis.

${ }^{22}$ Voir par exemple, SLIM Hédi, « Le modèle urbain romain et le problème de l'eau dans les confins du Sahel de la Basse Steppe », L'Afrique dans l'Occident romain (Ier siècle av. J.-C.-IVe siècle ap. J.-C.) », Actes du colloque de Rome (3-5 décembre 1987), Rome, EFR, 1990, p. 169-201.

${ }^{23}$ À ne pas traduire par modèle d'urbanisation ou d'urbanisme.

${ }^{24}$ Les « recettes » métallurgiques qui forment une grande part du Papyrus de Leyde ne constituent pas un contre-exemple, loin s'en faut. Les Alchimistes grecs. Tome I : Papyrus de LeydePapyrus de Stockholm - Recettes. Sous la direction de SAFFREY Henri Dominique. Texte établi et traduit par HALLEUX Robert (1981), Paris, Les Belles-Lettres, 2002; BERTHELOT Marcellin, RUELLE Charles-Émile, Collection des anciens alchimistes grecs, (1887-1888), Osnabrück, Zeller, 1967.

${ }_{25}$ DUNIKOWSKI Christophe et CABBOI Sandra, La sidérurgie chez les Sénons : les ateliers celtiques et gallo-romains des Clérimois (Yonne), Paris, éd. MSH, 1995 ; FOY Danièle, « Technologie, géographie, économie : les ateliers de verriers primaires et secondaires en Occident. Esquisse d'une évolution de l'Antiquité au Moyen Âge », dans NENNA Marie-Dominique (éd.) La Route du verre. Ateliers primaires et secondaires du second millénaire av. J.-C. au Moyen Âge. Lyon, Maison de l'Orient méditerranéen, 2000, p. 147-170 ; FERDIERE Alain, « La production de terres cuites architecturales en Gaule et dans l'Occident romain, à la lumière de l'exemple de la Lyonnaise et des cités du nord-est de l'Aquitaine : un artisanat rural de caractère domanial ? », Revue archéologique du Centre de la France, t. 51,2012 , p. $17-187$.

${ }^{26}$ PLATON, op. cit.

27 « La tendance tient à la nature même de l'évolution, les faits sont indissolublement liés au milieu dans lequel ils se produisent (...). La tendance a un caractère inévitable, prévisible, rectiligne ; 
elle pousse le silex tenu à la main à acquérir un manche, le ballot traîné sur deux perches à se munir de roues (...). », LEROIGOURHAN André, Évolution et techniques. L'homme et la Matière, Paris, Albin Michel, 1943, p. 27-28; et : " La nature même de la tendance est le mouvement général qui donne au milieu intérieur une adhésion de plus en plus étroite au milieu extérieur. Il est normal de retrouver partout cet 'élan' bergsonien (...). La tendance est propre au milieu intérieur, il ne peut y avoir de tendance du milieu extérieur : le vent ne propose pas à la maison un toit déterminé... », LEROI-GOURHAN A., Évolution et techniques. Milieux et Techniques, Paris, Albin Michel, 1945, p. 360 .

${ }^{28}$ Voir à ce propos les discussions provoquées dans l'espace économique francophone, par le Protocole de Londres (2000) destiné à simplifier le régime linguistique des brevets. Au cœur du débat, les spécificités d'énonciations, et donc de contenus, des apparats techniques en langue vernaculaire...

29 Le terme «culturel » n'est pas assimilable à «social »; il définit une composante de la structuration sociétale, au même titre que le politique, l'économique, le technique ou le religieux.

30 Concept venu de la phénoménologie, l'habitus désigne chez P. Bourdieu, des «structures prédisposées à fonctionner comme principes générateurs et organisateurs de pratiques et de représentations ", BOURDIEU Pierre, Le sens pratique, Paris, Éd. Minuit, 1980. Les groupes professionnels tendent à se constituer un habitus technique, dont l'existence précède l'habitus social et parfois s'assimile à lui lorsque le groupe en question adopte un profil d'invisibilité ou ne parvient pas à s'imposer dans la société.

${ }^{31}$ L'inverse est vrai. La patience d'Ikea pour s'implanter en Chine est remarquable. KIM Wall, "Ikea at last cracks China market, but success has meant adapting to local ways ", South China Morning Post, 01 September 2013. La classe moyenne chinoise s'intéresse difficilement au montage de meubles. D'autant qu'à côté d'un design très occidental, la conception des notices de montage l'est aussi. Une difficulté qu'une autre entreprise mondiale, Apple, a évitée en réduisant à quelques gestes la pratique de son smartphone, et en permettant, par le biais des " apps », l'adaptation aux habitudes culturelles de la clientèle.

32 GARÇON Anne-Françoise, "Réduire la mine en science ? Anatomie des De re metallica d'Agricola (1524-1556)», dans DUBOURG-GLATIGNY Pascal, VERIN Hélène, Réduire en Art. La technologie de la Renaissance aux Lumière, Paris, Éd. MSH, 2008, p. 317-336.

33 Jusqu'au moment où, de confession catholique, il refusa d'abjurer lorsque sa ville Schemnitz adopta la religion luthérienne.

34 De la fonte des mines, des fonderies, \&c. traduit de l'Allemand de Christophe-André SCHLUTTER. Tome premier, qui traite des essais des mines \& métaux, de l'affinage \& raffinage de l'argent, du départ de l'or, etc., le tout augmenté de plusieurs procédés \& observations, publié par M. HELLOT, de l'Académie Royale des Sciences, \& de la Société Royale de Londres, Paris, 1750.

35 GARÇon Anne-Françoise, "The Three States of Technology. An Historical Approach to a Thought Regime, XVIth-XX' ${ }^{\text {th }}$ Centuries », dans Michel FAUCHEUX et Joëlle FOREST (ed.), New Elements of Technology, Presses UTBM, Belfort-Montbéliard, 2012, p. 11-26.

${ }_{36}$ À titre d'exemple, je recommande la lecture du texte de LEIBNIZ G. W., Drôle de Pensée, touchant une nouvelle sorte de représentations (1675), disponible sur le site de l'Associations des Bibliophiles Universels (abu.cnam.fr).

37 Voyages métallurgiques. Recherches et Observations sur les mines \& forges de fer, la fabrication de l'acier, celle du ferblanc, \& plusieurs mines de charbon de terre, faites depuis l'année 1757 jusques \& compris 1769, en Allemagne, Suède, Norvège, Angleterre \& Écosse. Suivies d'un mémoire sur la circulation de l'air dans les mines et d'une notice de la jurisprudence des mines de charbon dans le Pays de Liège, la Province de Limbourg \& le Comté de Namur, par feu M. JARS, de l'Académie Royale des Sciences de Paris, de celle de Londres pour l'Encouragement des Arts \& Associé de l'Académie des Sciences, Belles-Lettres \& Arts de Lyon et publiés par M. G. JARS, Correspondant de l'Académie Royale des Sciences de Paris, \& associé à celle de Lyon, Lyon, 1774.

${ }^{8}$ GARÇON, 2012, op.cit., p. 83-99.

39 GARCON Anne-Françoise, «L'identité par la compétence: les ingénieurs d'industrie et la Société de l'Industrie Minérale, 18551875 », dans CARDOSO DE MATOS Ana et al. (dir.), Jogos de identitade professional : os engenheiros, a formaçao e a acçao, Lisbonne, Colibri, 2009, p. 403-419.

${ }^{40}$ GADAMER Hans Georg, Vérité et méthode. Les grandes lignes d'une herméneutique philosophique (1960), Paris, Seuil 1996.

${ }^{41}$ GARÇON, 2008, op. cit. p. 323-331.

${ }^{42}$ Pour ces auteurs, voir le site remarquable du Centre d'Histoire de la Renaissance de Tours, http://architectura.cesr.univtours.fr/, ainsi que LEMERLE Frédérique et PAUWELS Yves, Architectures de papier. La France et l'Europe (XVI $I_{-}-X V I I^{e}$ siècles), Brepols, Turnhout, 2013.

43 Traité de la Fonte des Mines par le feu du charbon de terre, ou traité de la construction et usage des fourneaux propres à la fonte et affinage des métaux et des minéraux, avec la manière de rendre ce charbon propre aux mêmes usages auxquels on emploie le charbon de bois, par M. de GENSSANE, de l'Académie Royale des Sciences de Montpellier, correspondant de celle de Paris \& concessionnaire des mines d'Alsace du Compté de Bourgogne, Vallat-la-Chapelle, 1770. 
44 Conceptualisée par Aristote et Cicéron, puis à l'époque moderne par Giambattista Vico, la topique est un espace de pensée dynamique, défini par des pôles notionnels qui en marquent les limites, au sein duquel s'élaborent les représentations mentales de l'objet en cours de conceptualisation. La détection des topiques en vigueur et de leur évolution est utile pour l'analyse historique des évolutions conceptuelles de la pensée technique.

$45 \mathrm{Nul}$ historien ou archéologue des techniques, y compris moimême, ne peut nier avoir recouru sans autres formes de procès aux planches du De re metallica ou de l'Encyclopédie, pour faire preuve dans un rapport de fouilles ou une interprétation de texte. Le problème n'est pas de s'appuyer sur ces ouvrages. Le problème est : sans autres formes de procès.

46 À l'inverse, le croisement histoire-archéologie a donné de belles études : GRANDEMANGE Jacques, Les mines d'argent du duché de Lorraine au XVIe siècle. Histoire et archéologie du Val de Lièpvre (Haut-Rhin), Paris Éd. MSH, 1991 ; RIETH, Éric, Le maître-gabarit, la tablette et le trébuchet essai sur la conception non-graphique des carènes du Moyen Âge au XXe siècle, Paris, CTHS, 1996 ; CHAPELOT Odette, Du projet au chantier, Paris, EHESS, 2001; FLAMBARD HERICHER Anne-Marie, Potiers et poteries du Bessin: histoire et archéologie d'un artisanat rural du XII ${ }^{e}$ au $X X^{e}$ siècle en Normandie, Caen, CRAHM, 2002 ; BRAUNSTEIN Philippe, Travail et entreprise au Moyen Âge, Bruxelles, De Boeck, 2003 ; ALLIOS Dominique, Le vilain et son pot, Rennes, PUR, 2004 ; ALEXANDRE-BIDON Danièle, Une archéologie du goût. Céramique et consommation (Moyen ÂgeTemps modernes), Paris, Picard, 2005 ; CHAPELOT Jean, CHAPELOT Odette, RIETH Bénédicte, Terres cuites architecturales médiévales et modernes en Île-de-France et dans les régions voisines, Caen, CRAHM, 2009; SERNA Virginie (dir.), Le Cher, Histoire et archéologie d'un cours d'eau, Tours, FERACF, 2013. La liste n'est pas exhaustive.

47 Citons, outre le désormais classique ANDRÉN Anders, Between Artifacts and Texts : Historical Archaeology in Global Perspective, New York, Springer, 1998, l'intéressant travail de SCHMIDT, Peter Ridgway, Historical Archaeology in Africa: Representation, Social Memory, and Oral traditions, Oxford, Altamira, 2006 ; GILCHRIST, Roberta, «Introduction: Scales and Woices in world Historical Archaeology ", World Archaeology, vol. $37, \mathrm{n}^{\circ} 3,2005$, p. 329-336 ; DILLIAN Carolyn D. et WHITE Carolyn L., Trade and Exchange: Archaeological Studies from History and Prehistory, New York, Springer, 2009.

${ }^{48}$ Le fameux : «Techniques, mot dont l'histoire n'est pas faite » par lequel débute le texte « Réflexions sur l'histoire des techniques », FEBVRE 1935, op. cit., passait sous silence la fondation entre 1932 et 1933 par Abel Rey de l'Institut d'Histoire et de Philosophie des Sciences et des Techniques, qui publia à partir de 1934 la revue Thalès. BRAUNSTEIN Jean-François, « Abel Rey et les débuts de l'Institut d'histoire des sciences et des tech- niques (1932-1940) », BITBOL Michel et GAYON Jean (dir.) L'Épistémologie française, $1830-1970$, Paris, PUF, 2006, p. 173191. Voir aussi l'important et tout récent GOUARNE Isabelle, L'introduction du marxisme en France. Philosoviétisme et sciences humaines, 1920-1939, Rennes, PUR, 2013.

49 Voici ce que Lucien Febvre écrivait au fil de ses « Réflexions sur l'histoire des techniques » : "L'histoire, écrivais-je en 1927, l'histoire ne s'édifiera pas par le labeur encyclopédique de quelques omniscients..., mais par 'l'effort courageux d'hommes de provenance, de culture et d'aptitudes diverses. Car ce n'est pas la même formation qu'il faut pour décrire le contenu d'une conscience chrétienne au XVIe siècle, le processus d'invention de la machine à vapeur ou la conception qu'avaient de la science les contemporains de Rousseau'. Une histoire comme celle des techniques, à plus forte raison. Et qui suppose le zèle convergent de techniciens curieux de leur technique et de son passé, mais qui peuvent être artisans, ingénieurs, chimistes, etc.; de savants connaissant l'histoire de leur science (...) ; d'historiens proprement dits enfin : historiens des civilisations à l'esprit synthétique; tous devant collaborer ensemble sous peine de voir leur travail demeurer insuffisant et sans portée. Mais collaborer, qu'est-ce ? Écrire chacun son chapitre, selon les vieilles méthodes communes ? A quoi bon ? (...) Collaboration, oui : autour de problèmes à étudier, et que chaque collaborateur doit étudier pour sa part, sans doute - mais avec obligation de confronter ensuite les résultats par lui obtenus dans les mêmes conditions par les coopérateurs du grand œuvre... ». Work in progress, de toute évidence.

50 Ainsi, Maurice Daumas (1910-1984) était chimiste de formation; Métallurgiste de haut vol et l'un des piliers de l'histoire des techniques aux États-Unis, Cyril Stanley Smith (1903-1992) avait participé au Manhattan Project (1942-1946) et fondé l'Institute for the Study of Metals à l'Université de Chicago; François Russo (1909-1998) était Polytechnicien; André Guillerme est ingénieur des travaux publics, Antoine Picon, ingénieur des Ponts \& Chaussées; Edmond Truffaut, Ingénieur, ancien directeur technique aux Aciéries de Paris et d'Outreau est l'auteur d'une thèse fondamentale Manganèse et acier. Contribution à l'histoire de la sidérurgie française. 1774-1906 (2000); Bernard Delaunay, ingénieur à Thalès, achève un doctorat sur la pensée technique de l'Académie royale des sciences, 1669-1740. Enfin, ce sont trois ingénieurs des mines, Armand Hatchuel, Pascal Le Masson et Benoît Weil qui ont initié en France, l'histoire de la conception technique... 